\title{
Mối quan hệ giữa hành vi thiếu văn minh của khách hàng và hành vi phá hoại dịch vụ của nhân viên trong ngành dịch vụ ở Việt Nam
}

\section{The relationship between customer mistreatment and employee's service sabotage in service industry in Vietnam}

\author{
Nguyễn Duy An ${ }^{1,2^{*}}$, Zhenyuan Wang ${ }^{1}$ \\ 1Đại học Sư phạm Hoa Đông, Thượng Hải, Trung Quốc \\ ${ }^{2}$ Trường Đại học Khoa học Xã hội và Nhân văn, ĐHQG-HCM, Việt Nam \\ *Tác giả liên hệ, Email: nguyenduyan93@gmail.com
}

THÔNG TIN

DOI: $10.46223 / \mathrm{HCMCOUJS}$. econ.vi.17.2.1617.2022

Ngày nhận: 15/03/2021

Ngày nhận lại: 22/05/2021

Duyệt đăng: 25/05/2021

\section{Tù khóa:}

hành vi thiếu văn minh của khách hàng; hành vi phá hoại dịch vụ của nhân viên; lý thuyết bảo tồn nguồn lực; tình trạng kiệt sức cảm xúc; sự ủng hộ từ tổ chức

\section{Keywords:}

customer mistreatment behavior; service sabotage behavior; conservation of resources theory; emotional exhaustiont; perceived organizational support

\section{TÓM TÁT}


organizational support moderates the mediation relationship negatively. Based on the conservation of resources theory, this study expands the relevant research on the employee's service sabotage in the context of Vietnam. In addition, this study also provides suggestions for reducing employee's service sabotage.

\section{1. Đặt vấn đề}

Trong một thời gian dài, nhiều doanh nghiệp cho rằng nhân viên ngành dịch vụ phải đề cao quan điểm "khách hàng là trên hết." Tuy nhiên, chính chuẩn mực quản lý kinh doanh này rất dễ gây ra những sai lệch trong nhận thức và làm tổn hại đến cảm xúc của nhân viên, có thể dẫn đến việc nhân viên có hành vi phá hoại dịch vụ, tạo ra tác động tiêu cực đến sản phẩm dịch vụ (Harris \& Ogbonna, 2006). Ngày nay, với sự bùng nổ của ngành dịch vụ, các công việc liên quan cũng dần phát triển, việc nhận thức không đầy đủ và phản ứng không kịp thời trong việc xử lý hành vi phá hoại dịch vụ ngày càng trở nên phổ biến. Có $75 \%$ nhân viên ngành dịch vụ thừa nhận họ đã thực hiện những hành vi không phù hợp trong quá trình cung cấp sản phẩm dịch vụ; $90 \%$ nhân viên cho rằng phá hoại dịch vụ là một hành vi hằng ngày (Harris \& Ogbonna, 2002). Ngoài ra, các hành vi thiếu văn minh của khách hàng (Customer Mistreatment) cũng tăng và nhân viên phục vụ thường gặp phải những "rắc rối hằng ngày” trong quá trình phục vụ khách hàng (Arnold \& Walsh, 2015; Sliter, Jex, Wolford, \& McInnerney, 2010; Sliter, Sliter, \& Jex, 2012; Wilson \& Holmvall, 2013). Những hành vi thiếu văn minh có vẻ không tác động lớn đến nhân viên; tuy nhiên, việc lặp đi lặp lại thường xuyên sẽ gây áp lực cho nhân viên và dẫn đến những phản ứng tiêu cực (Kim \& Qu, 2018; Sliter \& ctg., 2012).

Tại Việt Nam, sự căng thẳng giữa khách hàng và nhân viên đã được phản ánh khá rõ nét qua nhiều thông tin tiêu cực từ ngành dịch vụ như: hướng dẫn viên du lịch chèo kéo khách hàng mua sắm; nhân viên dọn phòng khách sạn dùng khăn mặt lau chùi nhà vệ sinh; khách hàng cự cãi, thậm chí còn bị khinh thường, áp bức đối với nhân viên phục vụ, ... Cụ thể là bài phóng sự "Góc khuất vệ sinh khách sạn: Dùng khăn tắm lau bồn cầu” trên báo Tuổi Trẻ tháng 07/2019 (Nguyen \& Thien Dieu, 2019); vụ tranh chấp giữa YouTuber Khoa Pug và Aroma Resort tháng 04/2019 (Quang Le, 2019); sự kiện bác sĩ gốc Việt bị "lôi” khỏi máy bay United Airlines năm 2017 (Thuy Linh, 2017), ...

Có rất ít nghiên cứu quan tâm đến ảnh hưởng của hành vi thiếu văn minh ở khách hàng đến tâm lý và hành vi của nhân viên phục vụ ở Việt Nam. Mục đích của bài nghiên cứu nhằm nhận diện, phân tích lý do nhân viên tham gia việc phá hoại dịch vụ đồng thời đưa ra biện pháp ngăn ngừa hành vi sai trái của họ. Từ đó, nghiên cứu sẽ gợi ý về logic lý thuyết và bằng chứng để giải thích tại sao nhân viên lại có những hành vi phá hoại dịch vụ trong quá trình cung cấp dịch vụ.

\section{Cơ sở lý thuyết và giả thuyết nghiên cứu}

\subsection{Anh hưởng của khách hàng có hành vi thiếu văn minh và hành vi phá hoại dịch vụ của nhân viên}

Hành vi thiếu văn minh của khách hàng là "hành vi lệch lạc cường độ thấp, gây tổn hại đến nhân viên với ý định không rõ ràng và vi phạm các chuẩn mực xã hội về sự tôn trọng và lịch sự lẫn nhau" (Andersson \& Pearson, 1999, tr. 454). Những hành vi thiếu văn minh không chỉ xuất hiện giữa các nhân viên với nhau mà còn xuất hiện khi giao tiếp với khách hàng như là hành động thiếu thân thiện và bất lịch sự (Kern \& Grandey, 2009; Sliter \& ctg., 2010). Hầu hết hành vi thiếu văn minh mà nhân viên phải đối mặt đến từ khách hàng nhiều hơn là từ đồng nghiệp. Có thể kể đến như: thái độ không tôn trọng hoặc to tiếng nhân viên; đưa ra các yêu cầu quá cao đối với dịch 
vụ, không quan tâm đến nguyên tắc và cảm xúc của nhân viên phục vụ, ... (Wang \& ctg., 2013).

Khái niệm Hành vi phá hoại dịch vụ (Service Sabotage) lần đầu tiên được đề xuất bởi Harris và Ogbonna (2002), dùng để chỉ những hành vi mà nhân viên cố tình gây ảnh hưởng xấu đến chất lượng dịch vụ khi cung cấp cho khách hàng. Có thể kể đến như: nhân viên cố tình bỏ qua hoặc phớt lờ các tiêu chuẩn dịch vụ do tổ chức đưa ra, thao túng tốc độ cung cấp dịch vụ; làm ngược lại các yêu cầu của khách hàng, thực hiện các hành vi tiêu cực, sử dụng ngôn từ thô lỗ, cố ý làm hỏng đồ đạc của khách hàng, tấn công khách hàng, ... Một số nghiên cứu nhận định rằng phá hoại dịch vụ là hành động trả đũa của nhân viên đối với khách hàng, trong đó bao gồm cả việc vi phạm các quy định của tổ chức dịch vụ, cũng như các hành vi thô lỗ và hạ thấp chất lượng dịch vụ của nhân viên (Skarlicki, Van Jaarsveld, \& Walker, 2008; Wang, Liao, Zhan, \& Shi, 2011).

Lý thuyết Bảo tồn Nguồn lực (Conservation of Resources Theory) cho rằng căng thẳng và áp lực có tác động xấu đến hành vi và cảm xúc của con người (Hobfoll, 1989). Con người khó kiểm soát được hành vi và cảm xúc khi chịu căng thẳng và áp lực (Bedi \& Schat, 2017). Các căng thẳng và áp lực từ các hành vi thiếu văn minh của khách hàng sẽ uy hiếp và làm tổn hại đến các nguồn lực nội tại sẵn có của nhân viên (Hur, Moon, \& Jun, 2016; Kim \& Qu, 2018; Torres, Van Niekerk, \& Orlowski, 2017). Mặt khác, đặc trưng của ngành dịch vụ là nhân viên phải tương tác với khách hàng hằng ngày, việc chịu đựng các hành vi thiếu văn minh từ khách hàng một cách bị động và thường xuyên chính là một áp lực lớn (Cho, Bonn, Han, \& Lee, 2016; Kern \& Grandey, 2009; Sliter \& ctg., 2010; Van Jaarsveld, Walker, \& Skarlicki, 2010). Khi đó, nhân viên sẽ nảy sinh tâm lý “ăn miếng trả miếng” đối với khách hàng có hành vi thiếu văn minh (Skarlicki \& ctg., 2008), dẫn đến hành vi phá hoại dịch vụ để giải tỏa áp lực (Spector \& Fox, 2002). Vì vậy, nghiên cứu đề xuất giả thuyết:

\section{H1: Hành vi thiếu văn minh có tác động thuận chiều đến hành vi phá hoại dịch vụ}

\subsection{Vai trò trung gian của tình trạng kiệt sức về cảm xúc trong ảnh hưởng của khách} hàng có hành vi thiếu văn minh và hành vi phá hoại dịch vụ của nhân viên

Kiệt sức về cảm xúc (Emotional exhaustion) là "cảm giác căng thẳng quá mức về mặt cảm xúc do công việc" (Maslach, Jackson, \& Leiter, 1997, tr. 194 ). Điều này xảy ra khi nhân viên cảm thấy mệt mỏi về thể chất hoặc kiệt quệ về tinh thần và cảm xúc (Wright \& Cropanzano, 1998).

Căn cứ vào lý thuyết Bảo tồn Nguồn lực, các nhân viên phải đầu tư thêm nguồn lực để điều chỉnh cảm xúc tiêu cực do những hành vi thiếu văn minh từ khách hàng gây ra (Sliter \& ctg., 2010; Van Jaarsveld \& ctg., 2010) sẽ khiến nguồn lực dành cho các nhiệm vụ trong công việc bị phân tán, từ đó sẽ giảm độ chính xác và chất lượng của công việc (Goldberg \& Grandey, 2007). Những nhân viên đang có cảm xúc tiêu cực sẽ khó kiểm soát được hành vi và thái độ khi làm việc (Denson, Pedersen, Friese, Hahm, \& Roberts, 2011) nên họ rất có khả năng tham gia vào việc phá hoại dịch vụ để giải phóng cảm xúc tiêu cực của họ (Wang \& ctg., 2011). Việc thường xuyên tiếp xúc với các khách hàng có hành vi thiếu văn minh không những trực tiếp dẫn đến các hành vi phá hoại dịch vụ, mà còn khiến nhân viên rơi vào trạng thái kiệt sức về cảm xúc, từ đó những nhân viên này có hành vi phá hoại dịch vụ để cân bằng lại nguồn lực của bản thân (Luo \& Bao, 2013). Vì vậy, nghiên cứu tiếp tục đề xuất giả thuyết:

H2: Kiệt sức về cảm xúc là nhân tố trung gian trong tác động hành vi thiếu văn minh đối với hành vi phá hoại dịch vu

\subsection{Vai trò điều tiết của sụ hỗ trọ' tù̀ tổ chức đối với ảnh hưởng của hành vi thiếu văn minh và hành vi phá họai dịch vụ thông qua kiệt sức về cảm xúc}

Sự hỗ trợ từ tổ chức (Perceived organizational support) được hiểu là mức độ nhận biết của cá nhân khi được tổ chức trân trọng và đánh giá cao, điều này nhằm để đổi lấy sự đóng góp, cống 
hiến của cá nhân dành cho với tổ chức (Eisenberger, Huntington, Hutchison, \& Sowa, 1986). Trong doanh nghiệp, sự hỗ trợ từ tổ chức là mức độ quan tâm đến hạnh phúc của công ty dành cho nhân viên, đây được xem như là "phần thưởng" khi các nhân viên hoàn thành công việc (Rhoades \& Eisenberger, 2002).

Dựa trên lý thuyết Bảo tồn Nguồn lực, thái độ và hành vi nhân viên phụ thuộc vào sự cân bằng giữa nguồn lực mà nhân viên tiêu thụ và nguồn lực thu được qua nhiều kênh khác nhau (Watts, Robertson, Winter, \& Leeson, 2013). Trong khi đó, hỗ trợ từ tổ chức có thể làm giảm ảnh hưởng giữa các tác nhân gây căng thẳng và phản ứng với căng thẳng (Lustig, Pessino, \& Scott, 2014; Wu, Changyang, \& Qin, 2019). Việc cải thiện nhân tố này có thể làm giảm tác hại của tình trạng kiệt sức trong công việc, trong đó có kiệt sức về cảm xúc (Watts \& ctg., 2013). Sự hỗ trợ từ tổ chức chính là một nguồn lực bổ sung quan trọng, giúp nhân viên cân bằng cảm xúc khi họ gặp phải các hành vi thiếu văn minh của khách hàng (Bacharach, Bamberger, \& Doveh, 2008; Sliter \& ctg., 2010; Van Jaarsveld \& ctg., 2010). Vì vậy, nghiên cứu đề xuất giả thuyết:

H3: Sụ hỗ trọ tù tổ chức điều tiết ngược chiều sự ảnh hương của hành vi thiếu văn minh của khách hàng đối với tình trạng kiệt sức về cảm xúc của nhân viên

Theo mối quan hệ giữa giả thuyết $\mathrm{H} 2$ và $\mathrm{H} 3$, bài nghiên cứu đề xuất thêm giả thuyết $\mathrm{H} 4$. Dưới các áp lực của các hành vi thiếu văn minh do khách hàng gây ra, sự hỗ trợ từ tổ chức sẽ bổ sung nguồn lực tinh thần làm giảm tình trạng kiệt sức về cảm xúc của nhân viên (Bacharach \& ctg., 2008; Sliter \& ctg., 2010; Van Jaarsveld \& ctg., 2010). Các nhân viên có mức độ cảm nhận hỗ trợ từ tổ chức cao sẽ điều chỉnh được thái độ và hành vi tiêu cực cao hơn các nhân viên khác, điều này giúp giảm tình trạng nhân viên tham gia vào các hành vi tiêu cực khi làm việc, điển hình là hành vi phá hoại dịch vụ (Denson \& ctg., 2011; Wang \& ctg., 2011). Vì vậy, nghiên cứu đề xuất thêm giả thuyết:

H4: Sụ hỗ trợ tù tổ chức điều tiết ngược chiều tác động của hành vi thiếu văn minh lên hành vi phá hoại dịch vu thông qua điều tiết ngược chiều sụ tác động của hành vi thiếu văn minh lên tình trạng kiệt sức về cảm xúc

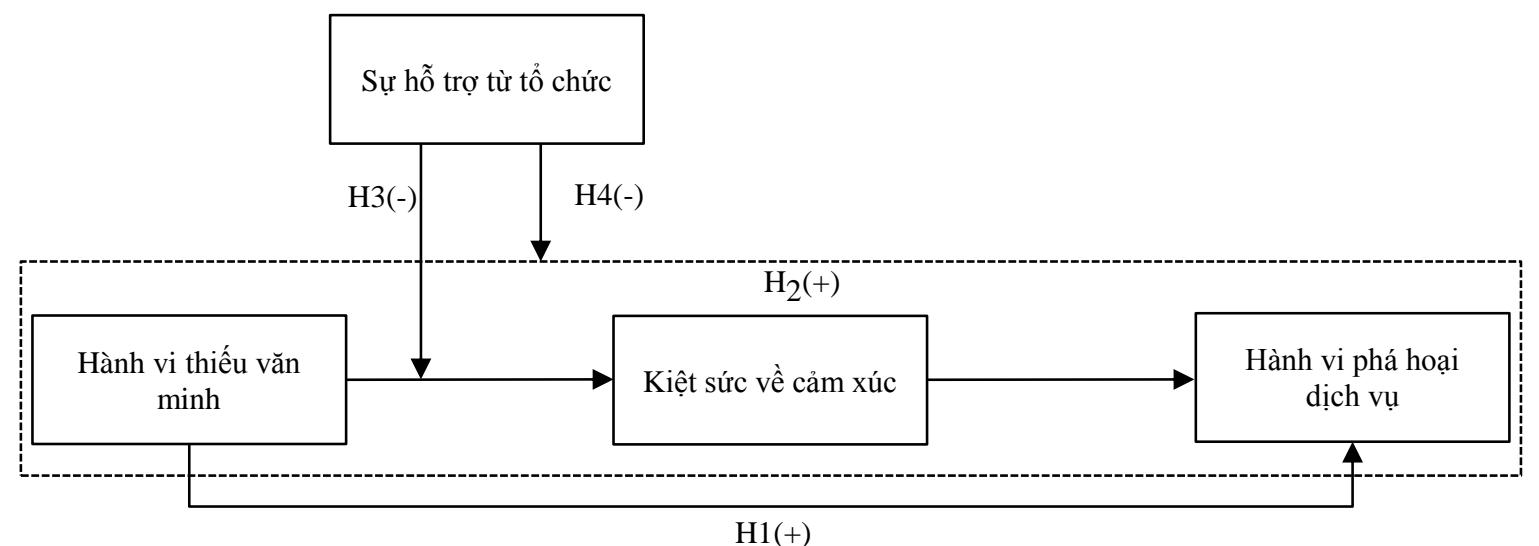

Hình 1. Mô hình nghiên cứu đề xuất

\section{Phương pháp nghiên cứu}

\subsection{Phwơng pháp thu thập dĩu liệu}

Hành vi phá hoại dịch vụ là hành vi cố tình gây ảnh hưởng xấu đến dịch vụ trong quá trình cung cấp dịch vụ cho khách hàng của nhân viên (Harris \& Ogbonna, 2002). Vì vậy, các nhân viên thường xuyên tiếp xúc với khách hàng chính là đối tượng phù hợp với nghiên cứu này. Nghiên cứu thu thập dữ liệu bằng phương pháp chọn mẫu định lượng: phi xác xuất - thuận tiện, thông qua 
hình thức phiếu khảo sát online từ Google Forms. Nhờ sự hỗ trợ của các nhân viên trong ngành nghề có đặc điểm công việc phù hợp với đối tượng nghiên cứu, như: ngành du lịch, chăm sóc khách hàng, spa, ... link phiếu khảo sát được chia sẻ trên Facebook, Zalo. Khảo sát thu được tổng cộng 655 bảng hỏi. Sau khi sàng lọc, nghiên cứu loại bỏ 206 bảng hỏi không phù hợp với mục đích nghiên cứu. Vì vậy, nghiên cứu chỉ sử dụng 449 (76.2\%) bảng hỏi hợp lệ.

Nghiên cứu sử dụng phần mềm SPSS 24.0 và AMOS 24.0 để phân tích dữ liệu, thông qua phân tích hồi quy thứ bậc trong SPSS 24.0 và phần mềm mở rộng PROCESS trong SPSS để kiểm tra các giả thuyết. Phân tích hồi quy thứ bậc nhằm kiểm tra các giả thuyết H1, H2, H3. Sau đó, nghiên cứu sử dụng phần mềm mở rộng PROCESS trong SPSS để tiến hành kiểm tra Bootstrap để kiểm tra giả thuyết H2, H4. Đặc điểm mẫu khảo sát chi tiết được trình bày cụ thể ở Bảng 1 .

\section{Bảng 1}

Thông tin mẫu nghiên cứu

\begin{tabular}{|c|c|c|c|}
\hline Mẫu n = 449 & Chỉ tiêu & Tần số & Tỷ lệ\% \\
\hline \multirow{2}{*}{ Giới tính } & Nam & 149 & $33.2 \%$ \\
\hline & Nữ & 300 & $66.8 \%$ \\
\hline \multirow{3}{*}{ Tuổi } & $18-25$ tuổi & 400 & $89.1 \%$ \\
\hline & $26-35$ tuổi & 45 & $10 \%$ \\
\hline & Trên 36 & 4 & $0.9 \%$ \\
\hline \multirow{4}{*}{ Kinh nghiệm làm việc } & Dưới 01 năm & 94 & $20.9 \%$ \\
\hline & 01 - 02 năm & 203 & $45.2 \%$ \\
\hline & 02 - 05 năm & 111 & $24.7 \%$ \\
\hline & 05 năm trở lên & 41 & $9.1 \%$ \\
\hline \multirow{2}{*}{ Tình trạng hôn nhân } & Độc thân & 384 & $85.5 \%$ \\
\hline & Đã kết hôn & 65 & $14.5 \%$ \\
\hline \multirow{4}{*}{ Trình độ học vấn } & THPT trở xuống & 36 & $8 \%$ \\
\hline & Trung cấp, cao đẳng & 116 & $25.8 \%$ \\
\hline & Đại học & 269 & $59.9 \%$ \\
\hline & Cao học trở lên & 28 & $6.2 \%$ \\
\hline \multirow{9}{*}{ Lĩnh vực công việc } & Du lịch lữ hành & 106 & $23.6 \%$ \\
\hline & Y tế & 12 & $2.7 \%$ \\
\hline & Nhà hàng, khách sạn & 102 & $22.7 \%$ \\
\hline & Ngân hàng, bảo hiểm & 14 & $3.1 \%$ \\
\hline & Giao thông vận tải & 11 & $2.4 \%$ \\
\hline & Bán lẻ & 14 & $3.1 \%$ \\
\hline & Bất động sản & 11 & $2.4 \%$ \\
\hline & Chăm sóc khách hàng & 93 & $20.7 \%$ \\
\hline & Thẩm mỹ, chăm sóc sắc đẹp & 86 & $19.2 \%$ \\
\hline
\end{tabular}

Nguồn: Kết quả phân tích từ SPSS 


\subsection{Thang do}

Để đảm bảo hiệu quả, nghiên cứu sử dụng các bảng hỏi được sử dụng trong những nghiên cứu trước đây, sau đó tiến hành dịch thuật và được các chuyên gia và nhân viên trong ngành dịch vụ góp ý sửa chữa nhằm phù hợp với môi trường Việt Nam. Bảng hỏi sử dụng thang đo Likert 5 bậc cho tất cả các biến.

Biến Hành vi thiếu văn minh của khách hàng (ký hiệu $\mathrm{CM}$ ), sử dụng thang đo Hành vi bắt nạt khách hàng do Wang và cộng sự (2013) đề xuất, gồm 18 câu trích, độ tin cậy Cronbach's $\alpha$ là 0.948 .

Biến Kiệt sức về cảm xúc (ký hiệu $\mathrm{EE}$ ), sử dụng thang đo Tình trạng Kiệt sức về cảm xúc trong bảng câu hỏi khảo sát của Maslach và cộng sự (1997) về tình trạng kiệt sức ở nơi làm việc MBI-GS (Maslach Burnout Inventory general survey), thang đo này gồm 05 câu trích, độ tin cậy Cronbach's $\alpha$ là 0.897.

Biến Hành vi phá hoại dịch vụ (ký hiệu SS), sử dụng thang đo Hành vi phá hoại dịch vụ của Harris và Ogbonna (2006), gồm 09 câu trích. Từ kết quả phân tích độ tin cậy Cronbach's $\alpha$, loại 02 biến quan sát (Tôi không bao giờ thể hiện bản thân trước mặt khách hàng; Ở công ty, khách hàng sẽ được đối xử rất tốt), độ tin cậy Cronbach's $\alpha$ là 0.848 .

Biến Sự hỗ trợ từ tổ chức (ký hiệu POS), sử dụng Eisenberger và cộng sự (1986), gồm 08 câu trích. Từ kết quả phân tích độ tin cậy Cronbach's Alpha, loại 01 biến quan sát (Ngay cả khi tôi cố gắng làm tốt nhất có thể công việc của mình, công ty cũng không quan tâm), độ tin cậy Cronbach's $\alpha$ là 0.923 .

Biến Kiểm soát: nghiên cứu sử dụng các biến nhân khẩu học phổ biến trong hành vi của tổ chức như giới tính, lứa tuổi, kinh nghiệm làm việc, tình trạng hôn nhân, trình độ học vấn, lĩnh vực công việc.

\section{Kết quả nghiên cứu}

\subsection{Phân tích CFA}

Trong nghiên cứu này, phân tích CFA tuân thủ theo kiến nghị của Hui, Lee, và Rousseau (2004) tiến hành gộp biến đối với việc xây dựng nhân tố có nhiều hơn 03 biến quan sát. Theo cách này, mỗi nhân tố chỉ có 03 biến quan sát, căn cứ vào kết quả phân tích nhân tố, gộp biến có hệ số tải nhân tố cao nhất và biến có hệ số tải nhân tố thấp nhất thành một biến bằng cách lấy hệ số trung bình của hai biến, gộp biến có hệ số tải nhân tố cao thứ hai và biến có hệ số tải nhân tố thấp thứ hai thành một biến, ... Tiến hành tương tự như vậy cho đến khi chỉ còn 03 biến quan sát trong một nhân tố.

Căn cứ vào Bảng 2, các chỉ số của mô hình bao gồm 04 nhân tố (mô hình đề xuất của nghiên cứu) với các giá trị $x^{2}=102.803, x^{2} / d f=2.142, \mathrm{GFI}=0.963, \mathrm{TLI}=0.982, \mathrm{CFI}=0.987$ đều cao hơn giá trị công nhận 0.900 ; RMSEA là 0.050 , thấp hơn giá trị công nhận chung là 0.080 , cho thấy dữ liệu mô hình giả định phù hợp.

So sánh mô hình 04 nhân tố (mô hình đề xuất) với ba mô hình thay thế khác, mô hình 04 nhân tố $\left(x^{2}=102.803, x^{2} / d f=2.142\right)$ có các chỉ số phù hợp hơn so với ba mô hình còn lại. Mô hình 03 nhân tố $\left(x^{2}=798.280, x^{2} / d f=15.653\right)$ kết hợp nhân tố Hành vi thiếu văn minh và nhân tố Kiệt sức về cảm xúc. Mô hình 02 nhân tố $\left(x^{2}=1851.717, x^{2} / d f=34.938\right)$ kết hợp nhân tố Hành vi thiếu văn minh, nhân tố Kiệt sức về cảm xúc và nhân tố Sự hỗ trợ từ tổ chức. Mô hình 01 nhân tố $\left(x^{2}=2254.068, x^{2} / d f=41.742\right)$ kết hợp tất cả các nhân tố. Như vậy, có thể kết luận rằng mô hình 04 nhân tố đáng tin cậy và phù hợp với dữ liệu khảo sát. 


\section{Bảng 2}

Kết quả phân tích CFA

\begin{tabular}{|l|l|c|c|c|c|c|c|c|c|}
\hline \multicolumn{1}{|c|}{ Mô hình } & \multicolumn{1}{|c|}{ Nhân tố } & $\boldsymbol{x}^{\mathbf{2}}$ & $\boldsymbol{d} \boldsymbol{f}$ & $\frac{\boldsymbol{x}^{\mathbf{2}}}{\boldsymbol{d f}}$ & GFI & CFI & TLI & NFI & RMSEA \\
\hline $\begin{array}{l}\text { Mô hình 04 } \\
\text { nhân tố (Mố } \\
\text { hình đề xuât) }\end{array}$ & $\begin{array}{l}\text { CM, EE, } \\
\text { POS, SS }\end{array}$ & 102.803 & 48 & 2.142 & 0.963 & 0.987 & 0.982 & 0.976 & 0.050 \\
\hline $\begin{array}{l}\text { Mô hình 03 } \\
\text { nhân tố }\end{array}$ & $\begin{array}{l}\text { CM+EE, } \\
\text { POS, SS }\end{array}$ & 798.280 & 51 & 15.653 & 0.750 & 0.822 & 0.769 & 0.812 & 0.181 \\
\hline $\begin{array}{l}\text { Mô hình 02 } \\
\text { nhân tố }\end{array}$ & $\begin{array}{l}\text { CM+EE+P } \\
\text { OS, SS }\end{array}$ & $1,851.717$ & 53 & 34.938 & 0.573 & 0.571 & 0.465 & 0.565 & 0.275 \\
\hline $\begin{array}{l}\text { Mô hình 01 } \\
\text { nhân tố }\end{array}$ & $\begin{array}{l}\text { CM+EE+P } \\
\text { OS+SS }\end{array}$ & $2,254.068$ & 54 & 41.742 & 0.497 & 0.475 & 0.368 & 0.470 & 0.302 \\
\hline
\end{tabular}

Ghi chú: CM: Hành vi thiếu văn minh; EE: Kiệt sức về cảm xúc; POS: Sự hỗ trợ từ tổ chức; SS: Hành vi phá hoại dịch vụ; + nghĩa là 02 nhân tố gộp lại thành 01 nhân tố

Nguồn: Kết quả phân tích từ AMOS

\subsection{Phân tích tương quan}

Từ Bảng 3 cho thấy Hành vi thiếu văn minh của khách hàng, Hành vi phá hoại dịch vụ của nhân viên và tình trạng Kiệt sức về cảm xúc của nhân viên tương quan thuận chiều, hệ số tương quan lần lượt là $r=0.394, \mathrm{p}<0.001 ; r=0.478, \mathrm{p}<0.001$. Sự hỗ trợ từ tổ chức và Hành vi phá hoại dịch vụ, Hành vi thiếu văn minh của khách hàng, tình trạng Kiệt sức về cảm xúc tương quan ngược chiều, hệ số tương quan lần lượt là $r=-0.279, \mathrm{p}<0.001 ; r=-0.203, \mathrm{p}<0.001 ; r=-0.359$, $\mathrm{p}<0.001$. Kết quả này sẽ cung cấp cơ sở cho việc kiểm định các giả thuyết.

\section{Bảng 3}

Hệ số tương quan, giá trị trung bình $(\mathrm{M})$, độ lệch chuẩn $(\mathrm{SD})$ giữa các biến

\begin{tabular}{|c|c|c|c|c|c|c|c|c|c|c|}
\hline & $\mathbf{1}$ & $\mathbf{2}$ & $\mathbf{3}$ & $\mathbf{4}$ & $\mathbf{5}$ & $\mathbf{6}$ & $\mathbf{7}$ & $\mathbf{8}$ & $\mathbf{9}$ & $\mathbf{1 0}$ \\
\hline 1. Giới tính & 1 & & & & & & & & & \\
\hline 2. Tuổi & $.130^{* *}$ & 1 & & & & & & & & \\
\hline $\begin{array}{c}\text { 3. Kinh nghiệm } \\
\text { làm việc }\end{array}$ & .092 & $.346^{* * *}$ & 1 & & & & & & & \\
\hline $\begin{array}{c}\text { 4. Tình trạng } \\
\text { hôn nhân }\end{array}$ & -.035 & $.228^{* * *}$ & $.350^{* * *}$ & 1 & & & & & & \\
\hline $\begin{array}{c}\text { 5. Tình trạng } \\
\text { học vấn }\end{array}$ & .047 & $.182^{* * *}$ & $.245^{* * *}$ & $.178^{* * *}$ & 1 & & & & & \\
\hline $\begin{array}{c}\text { 6. Lĩnh vực } \\
\text { công viẹ̣c }\end{array}$ & $-.246^{* * *}$ & $-.185^{* * *}$ & -.062 & -.034 & $-.312^{* * *}$ & 1 & & & & \\
\hline 7. SS & .075 & $-.126^{* *}$ & .034 & .083 & -.059 & -.038 & 1 & & & \\
\hline 8. CM & $.096^{*}$ & .081 & -.073 & -.051 & -.028 & $-.162^{* *}$ & $.394^{* * *}$ & 1 & & \\
\hline
\end{tabular}




\begin{tabular}{|c|c|c|c|c|c|c|c|c|c|c|}
\hline & $\mathbf{1}$ & $\mathbf{2}$ & $\mathbf{3}$ & $\mathbf{4}$ & $\mathbf{5}$ & $\mathbf{6}$ & $\mathbf{7}$ & $\mathbf{8}$ & $\mathbf{9}$ & $\mathbf{1 0}$ \\
\hline $9 . \mathrm{EE}$ & -.003 & -.057 & -.024 & $.102^{*}$ & -.075 & $.097^{*}$ & $.541^{* * *}$ & $.478^{* * *}$ & 1 & \\
\hline $10 . \mathrm{POS}$ & -.077 & -.056 & -.015 & -.055 & .025 & $.095^{*}$ & $-.279^{* * *}$ & $-.203^{* * *}$ & $-.359^{* * *}$ & 1 \\
\hline M & .33 & 22.51 & 2.22 & .14 & 2.64 & 5.17 & 2.30 & 2.90 & 2.58 & 3.639 \\
\hline SD & .417 & 3.763 & .880 & .352 & .718 & 2.923 & .834 & .833 & .959 & .886 \\
\hline
\end{tabular}

Ghi chú: $* * * \mathrm{p}<0.001 ; * * \mathrm{p}<0.01 ; * \mathrm{p}<0.05$

Nguồn: Kết quả phân tích từ SPSS

\subsection{Tác động của hành vi thiếu văn minh đến hành vi phá hoại dịch vụ}

Thông qua kết quả phân tích từ Bảng 4 , hành vi thiếu văn minh tác động thuận chiều đến hành vi phá hoại dịch vụ, $\beta=0.418, p=0.000<0.001$ (Bảng 4, Mô hình 4), chấp nhận giả thuyết H1.

\section{Bảng 4}

Kết quả phân tích hồi quy thứ bậc

\begin{tabular}{|c|c|c|c|c|c|c|c|}
\hline \multirow[t]{2}{*}{ Biến } & \multicolumn{2}{|c|}{ EE } & \multicolumn{3}{|c|}{ SS } & \multicolumn{2}{|c|}{$\mathbf{E E}$} \\
\hline & Mô hình 1 & Mô hình 2 & Mô hình 3 & Mô hình 4 & Mô hình 5 & Mô hình 6 & Mô hình 7 \\
\hline $\mathrm{CM}$ & & $.520 * * *$ & & $.418^{* * *}$ & $.189 * * *$ & $.467 * * *$ & $.464 * * *$ \\
\hline $\mathrm{EE}$ & & & & & $.440 * * *$ & & \\
\hline POS & & & & & & $-.280 * * *$ & $-.272 * * *$ \\
\hline CM X POS & & & & & & & $-.090 *$ \\
\hline Giới tính & .074 & .013 & .176 & .127 & .121 & -.014 & -.011 \\
\hline Tuổi & -.015 & $-.028 *$ & $-.049 * * *$ & $-.059 * * *$ & $-.047 * * *$ & $-.029 * *$ & $-.029 * *$ \\
\hline $\begin{array}{l}\text { Kinh nghiệm } \\
\text { làm việc }\end{array}$ & -.044 & .011 & .075 & $.119 *$ & $.114^{*}$ & .010 & .011 \\
\hline $\begin{array}{l}\text { Tình trạng } \\
\text { hôn nhân }\end{array}$ & $.403 * *$ & $.450 * * *$ & $.336 * *$ & $.373 * *$ & .175 & $.395 * *$ & $.416 * * *$ \\
\hline $\begin{array}{l}\text { Trình độ học } \\
\text { vấn }\end{array}$ & -.080 & -.030 & -.125 & -.084 & -.071 & -.007 & -.001 \\
\hline $\begin{array}{l}\text { Lĩnh vực } \\
\text { công việc }\end{array}$ & .027 & $.056 * * *$ & -.024 & -.002 & -.026 & $.062 * * *$ & $.050 * *$ \\
\hline $\mathrm{R}^{2}$ & .031 & .288 & .049 & .215 & .353 & .362 & .372 \\
\hline $\mathrm{R}^{2}$ hiệu chỉnh & .017 & .277 & .036 & .203 & .342 & .351 & .359 \\
\hline$\Delta R^{2}$ & .031 & .258 & .049 & .166 & .138 & .074 & .009 \\
\hline $\mathrm{F}$ & 2.319 & 25.489 & 3.823 & 17.295 & 30.056 & 31.251 & 28.845 \\
\hline
\end{tabular}

Ghi chú: *** $\mathrm{p}<0.001 ; * * \mathrm{p}<0.01 ; * \mathrm{p}<0.05$

Nguồn: Kết quả phân tích từ SPSS

4.4. Vai trò trung gian của tình trạng kiệt sức về cảm xúc trong mối quan hệ giữa hành vi thiếu văn minh của khách hàng và hành vi phá hoại dịch vụ của nhân viên

Hành vi thiếu văn minh có tác động thuận chiều đến Kiệt sức về cảm xúc, $\beta=0.520, \mathrm{p}=$ $0.000<0.001$ (Bảng 4, Mô hình 2). Kiệt sức về cảm xúc tác động thuận chiều đến Hành vi phá 
hoại dịch vụ, $\beta=0.440, \mathrm{p}=0.000<0.001$ (Bảng 4 , Mô hình 5 ). Từ Mô hình 5 cũng có thể thấy rằng sau khi thêm biến Kiệt sức về cảm xúc, so với Mô hình 4, hệ số hồi quy về Hành vi thiếu văn minh của khách hàng và Hành vi phá hoại dịch vụ của nhân viên tuy giảm nhưng vẫn có ý nghĩa tương quan, $\beta=0.189, \mathrm{p}=0.000<0.001$.

Tiến hành kiểm tra Bootstrap trên phần mềm mở rộng PROCESS trong SPSS. Ảnh hưởng trung gian của tình trạng Kiệt sức về cảm xúc đạt đến mức ý nghĩa $\beta=0.229$, khoảng $95 \%$ độ tin cậy [0.172; 0.295], không bao gồm 0 , như vậy tác dụng trung gian của tình trạng Kiệt sức về cảm xúc có ý nghĩa. Đồng thời, tác động trực tiếp của Hành vi thiếu văn minh đối với hành vi phá hoại dịch vụ vẫn có ý nghĩa, $\beta=0.188$, khoảng 95\% độ tin cậy [0.098; 0.278$]$, không bao gồm 0 , giả thuyết $\mathrm{H} 2$ được chấp nhận.

\subsection{Anh hưởng điều tiết của nhân tố hỗ trọ' tù̀ tổ chức trong sụ ảnh hưởng của hành vi} thiếu văn minh đối với tình trạng kiệt sức về cảm xúc

Biến hệ số nhân giữa Hành vi thiếu văn minh của khách hàng và Sự hỗ trợ từ tổ chức tác động ngược chiều lên tình trạng Kiệt sức về cảm xúc của nhân viên, $\beta=-0.090, \mathrm{p}=0.01<0.05$ (Bảng 4, Mô hình 7), cho thấy Sự hỗ trợ từ tổ chức điều tiết ngược chiều sự ảnh hưởng của Hành vi thiếu văn minh của khách hàng đối với tình trạng Kiệt sức về cảm xúc của nhân viên, giả thuyết H3 được chấp nhận.

Hình 2 mô tả tác dụng điều tiết của nhân tố Sự hỗ trợ từ tổ chức trong ảnh hưởng của Hành vi thiếu văn minh đối với tình trạng Kiệt sức về cảm xúc. Từ Hình 2 có thể thấy rằng nếu nhân viên có mức độ cảm nhận hỗ trợ từ tổ chức thấp, độ dốc thể hiện sự ảnh hưởng của Hành vi thiếu văn minh của khách hàng đối với tình trạng Kiệt sức về cảm xúc của nhân viên lớn hơn nhân viên có mức độ cảm nhận hỗ trợ từ tổ chức cao. Như vậy, nhân viên có mức độ cảm nhận hỗ trợ từ tổ chức thấp sẽ chịu tác động của Hành vi thiếu văn minh của khách hàng dẫn đến tình trạng Kiệt sức mạnh hơn nhân viên có Sự hỗ trợ từ tổ chức cao.

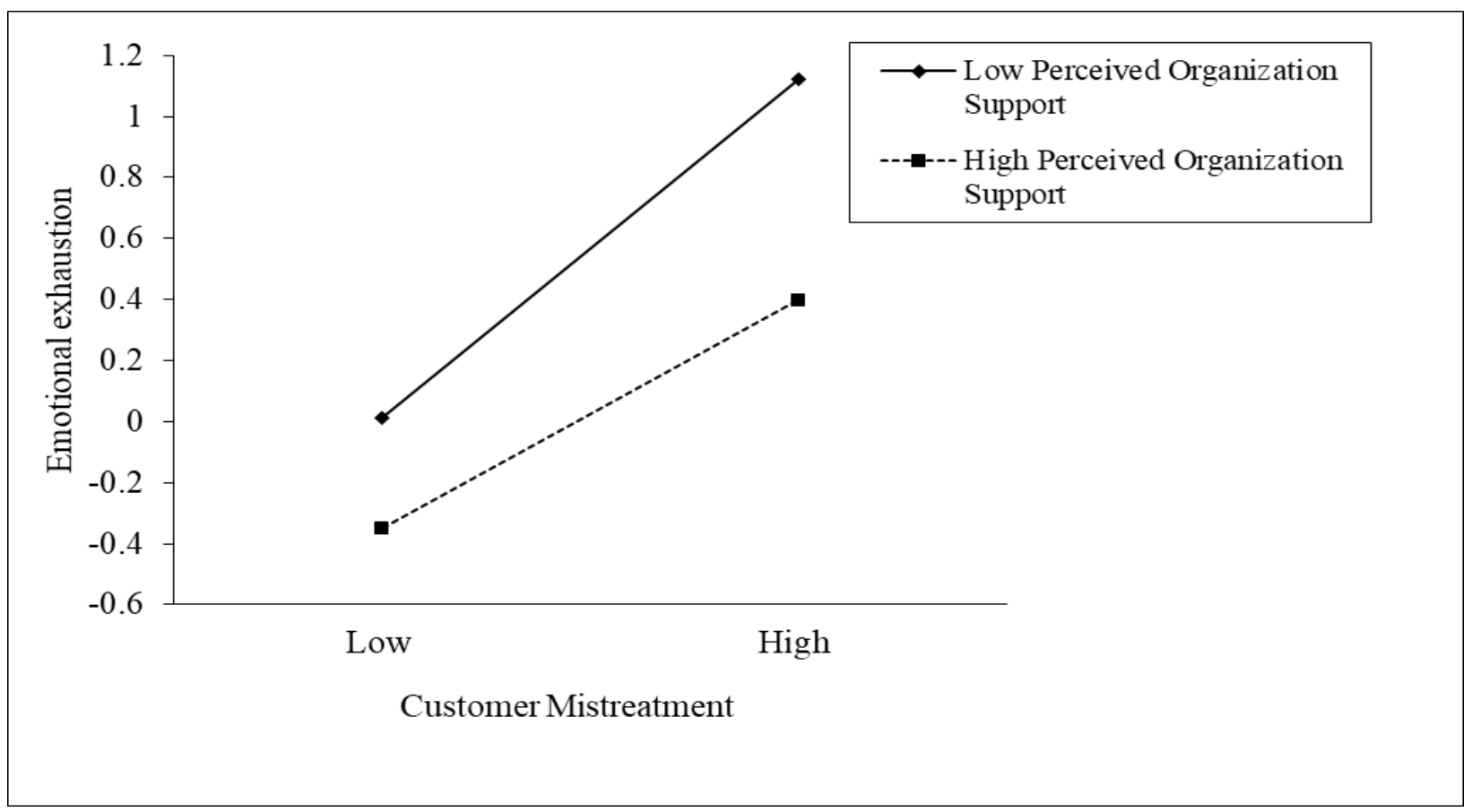

Hình 2. Ảnh hưởng điều tiết Sự hỗ trợ từ tổ chức đối với ảnh hưởng của Hành vi thiếu văn minh đối với Hành vi phá hoại dịch vụ

Nguồn: Kết quả phân tích của nghiên cứu 


\subsection{Tác động điều tiết của sụ hỗ trợ tù̀ tổ chức trong ảnh hưởng của hành vi thiếu văn minh lên hành vi phá hoại dịch vụ thông qua tình trạng kiệt sức về cảm xúc của nhân viên}

Trên cơ sở giả thuyết H2, H3 được chấp nhận, nghiên cứu tiến hành kiểm tra Bootstrap trên phần mềm mở rộng PROCESS trong SPSS. Theo kết quả phân tích từ Bảng 5 , khi Sự hỗ trợ từ tổ chức thấp, ảnh hưởng của Hành vi thiếu văn minh lên Hành vi phá hoại dịch vụ thông qua Kiệt sức về cảm xúc có ý nghĩa, $\beta=0.243$, khoảng 95\% độ tin cậy [0.174; 0.321$]$, không bao gồm 0 ; khi Sự hỗ trợ từ tổ chức cao, ảnh hưởng của Hành vi thiếu văn minh lên Hành vi phá hoại dịch vụ thông qua việc Kiệt sức về cảm xúc có ý nghĩa, $\beta=0.164$, khoảng 95\% độ tin cậy [0.108; 0.231], không bao gồm 0 ; chênh lệch giữa Sự hỗ trợ từ tổ chức cao và thấp đều có ý nghĩa, $\beta=$ 0.039 , khoảng $95 \%$ độ tin cậy [-0.076; -0.009], không bao gồm 0 . Như vậy, Sự hỗ trợ từ tổ chức điều tiết ngược chiều sự ảnh hưởng của hành vi thiếu văn minh lên hành vi phá hoại dịch vụ thông qua kiệt sức về cảm xúc, giả thuyết $\mathrm{H} 4$ được chấp nhận.

\section{Bảng 5}

Kết quả kiểm tra Bootstrap của Mô hình biến trung gian Mediator

\begin{tabular}{|l|c|c|c|c|}
\hline \multicolumn{1}{|c|}{ Biến điều tiết } & Tác dụng ảnh hưởng gián tiếp & SE & \multicolumn{2}{|c|}{ 95\% khoảng tin cậy } \\
\hline & & & Boot LLCI & Boot ULCI \\
\hline Sự hỗ trợ từ tổ chức thấp & .243 & .036 & .174 & .321 \\
\hline Sự hỗ trợ từ tồ chức cao & .164 & .031 & .108 & .231 \\
\hline $\begin{array}{l}\text { Chênh lệ̂ch giữa Sự hỗ } \\
\text { trợ từ tố chức cao và thấp }\end{array}$ & -.039 & .017 & -.076 & -.009 \\
\hline
\end{tabular}

Nguồn: Kết quả phân tích từ SPSS

\section{Kết luận và ý nghĩa của nghiên cứu}

\subsection{Kết luận}

Hành vi ứng xử thiếu văn minh của khách hàng sẽ khiến các nhân viên dễ tham gia vào hành vi phá hoại dịch vụ (Giả thuyết $\mathrm{H} 1)$. Hành vi thiếu văn minh từ khách hàng sẽ khiến các nhân viên rơi vào tình trạng kiệt sức về cảm xúc, từ đó các nhân viên này sẽ tham gia vào các hành vi phá hoại dịch vụ (Giả thuyết $\mathrm{H} 2$ ). Kết quả này cũng tương đồng với các nghiên cứu trước (Hur \& ctg., 2016; Luo \& Bao, 2013; Van Jaarsveld \& ctg., 2010; Wang \& ctg., 2013).

Sự hỗ trợ từ tổ chức có vai trò làm giảm tác động ảnh hưởng của hành vi thiếu văn minh của khách hàng đối với tình trạng kiệt sức về cảm xúc của nhân viên (Giả thuyết H3). Sự hỗ trợ từ tổ chức điều tiết ngược chiều sự ảnh hưởng của hành vi thiếu văn minh đối với hành vi phá hoại dịch vụ thông qua điều tiết ngược chiều sự ảnh hưởng của hành vi thiếu văn minh của khách hàng đối với tình trạng kiệt sức về cảm xúc của nhân viên (Giả thuyết $\mathrm{H} 4$ ). Kết quả này cũng tương đồng với các nghiên cứu trước, sự hỗ trợ từ tổ chức có vai trò điều tiết ảnh hưởng của tác nhân gây căng thẳng và phản ứng của nhân viên (Lustig \& ctg., 2014).

\section{2. Ý nghĩa của nghiên cứu}

Nghiên cứu này đã mở rộng nghiên cứu về cơ chế ảnh hưởng của hành vi thiếu văn minh của khách hàng đến tâm lý và hành vi của nhân viên trong ngành dịch vụ ở Việt Nam, giúp tìm hiểu cơ chế của hành vi phá hoại dịch vụ của nhân viên từ các góc độ lý thuyết Bảo tồn Nguồn lực tại Việt Nam. Nghiên cứu đã liên kết thành công các hành vi thiếu văn minh của khách hàng với 
các hành vi phá hoại dịch vụ của nhân viên, cho thấy quy luật: Hành vi thiếu văn minh của khách hàng sẽ dẫn đến hành vi phá hoại dịch vụ của nhân viên tại Việt Nam. Nghiên cứu này còn đưa ra biến số trung gian của các hành vi thiếu văn minh tại nơi làm việc ảnh hưởng đến các hành vi phá hoại dịch vụ, đó là kiệt sức về cảm xúc.

Ngoài ra, kết quả nghiên cứu này cũng nhấn mạnh tầm quan trọng của Sự hỗ trợ từ tổ chức đối với vai trò điều tiết trong mối liên kêt: Hành vi thiếu văn minh - Kiệt sức về cảm xúc - Hành vi phá hoại dịch vụ. Trong các nghiên cứu trước đây, có rất ít trường hợp chú ý đến các nhân tố làm giảm hành vi phá hoại dịch vụ của nhân viên (Wang \& ctg., 2011). Kết quả của nghiên cứu này chứng minh rằng sự hỗ trợ từ tổ chức là có ý nghĩa trong việc làm giảm các cảm xúc và hành vi phá hoại dịch vụ của nhân viên do các hành vi thiếu văn minh của khách hàng gây ra.

\subsection{Khuyến nghị về thục tiễn}

Các nhà quản lý nên nhận ra sự tồn tại của hành vi thiếu văn minh của khách hàng để tìm cách giảm thiểu ảnh hưởng của nó bằng cách: xây dựng quy trình công việc và quy định nội bộ đối với nhân viên nhằm "hạn chế" tác động xấu khi nhân viên gặp phải khách hàng thực hiện các hành vi thiếu văn minh; sử dụng các chiến lược tuyên truyền để củng cố hành vi văn minh của khách hàng, tăng cường ủy quyền cho nhân viên nhằm giảm tỷ lệ khách hàng khiếu nại; đồng thời tạo ra môi trường phục vụ chu đáo và hài hòa để thúc đẩy các hành vi văn minh; cho phép nhân viên từ chối cung cấp dịch vụ khi gặp phải một số hành vi quá đáng từ khách hàng.

Doanh nghiệp nên quan tâm đến tâm trạng và cảm xúc của nhân viên, cung cấp các giải pháp và hỗ trợ tinh thần kịp thời khi nhân viên tiếp xúc với khách hàng thiếu văn minh. Doanh nghiệp cần chủ động và thường xuyên trao đổi với nhân viên, thấu hiểu khó khăn của những nhân viên này khi gặp phải trong công việc để kịp thời giúp đỡ và hỗ trợ trong trường hợp cần thiết; xây dựng chương trình huấn luyện về tâm lý và kỹ năng cho nhân viên để họ biết cách ứng phó với các hành vi thiếu văn minh từ khách hàng, ...

\subsection{Hạn chế và gợi ý cho nghiên cứu tiếp theo}

Đầu tiên, đó là hiện tượng đa cộng tuyến (multi-collinearity) giữa các biến với nhau, tuy rằng các chỉ số phân tích $\mathrm{CFA}$ đã chứng minh rằng giữa các nhân tố có tính phân biệt, đa cộng tuyến không quá nghiêm trọng. Tuy nghiên, dữ liệu nghiên cứu được thu thập từ một mốc thời gian nên vẫn còn hạn chế. Các nghiên cứu tiếp theo có thể thu thập số liệu từ nhiều mốc thời gian khác nhau, hoặc dùng các phương pháp khác để giảm hiện tượng đa cộng tuyến. Ngoài ra, còn có thể khảo sát thêm những nhân tố khác đã sự hưởng đến hành vi phá hoại dịch vụ của nhân viên như thế nào, ví dụ: đồng nghiệp có hành vi thiếu văn minh, lãnh đạo có hành vi bắt nạt nhân viên, ...

Một hạn chế khác là dữ liệu nghiên cứu thu thập từ nhiều ngành nghề, nghiên cứu tiếp theo có thể tập trung nghiên cứu một ngành dịch vụ nhất định để khái quát sâu sắc hơn quy luật dẫn đến các hành vi phá hoại dịch vụ của nhân viên, ví dụ: ngành nhà hàng khách sạn, ...

\section{Tài liệu tham khảo}

Andersson, L. M., \& Pearson, C. M. (1999). Tit for tat? The spiraling effect of incivility in the workplace. The Academy of Management Review, 24(3), 452-471.

Arnold, K. A., \& Walsh, M. M. (2015). Customer incivility and employee well-being: Testing the moderating effects of meaning, perspective taking and transformational leadership. Work and Stress, 29(4), 362-378. 
Bacharach, S. B., Bamberger, P. A., \& Doveh, E. (2008). Firefighters, critical incidents, and drinking to cope: The adequacy of unit-level performance resources as a source of vulnerability and protection. Journal of Applied Psychology, 93(1), 155-169.

Bedi, A., \& Schat, A. C. H. (2017). Employee revenge against uncivil customers. Journal of Services Marketing, 31(6), 636-649.

Cho, M., Bonn, M. A., Han, S. J., \& Lee, K. H. (2016). Workplace incivility and its effect upon restaurant frontline service employee emotions and service performance. International Journal of Contemporary Hospitality Management, 28(12), 2888-2912.

Denson, T. F., Pedersen, W. C., Friese, M., Hahm, A., \& Roberts, L. (2011). Understanding impulsive aggression: Angry rumination and reduced self-control capacity are mechanisms underlying the provocation-aggression relationship. Personality and Social Psychology Bulletin, 37(6), 850-862.

Eisenberger, R., Huntington, R., Hutchison, S., \& Sowa, D. (1986). Perceived organizational support. Journal of Applied Psychology, 71(3), 500-507.

Goldberg, L. S., \& Grandey, A. A. (2007). Display rules versus display autonomy: Emotion regulation, emotional exhaustion, and task performance in a call center simulation. Journal of Occupational Health Psychology, 12(3), 301-318.

Harris, L. C., \& Ogbonna, E. (2002). Exploring service sabotage: The antecedents, types and consequences of frontline, deviant, antiservice behaviors. Journal of Service Research, 4(3), 163-183.

Harris, L. C., \& Ogbonna, E. (2006). Service sabotage: A study of antecedents and consequences. Journal of the Academy of Marketing Science, 34(4), 543-558.

Hobfoll, S. E. (1989). Conservation of resources: A new attempt at conceptualizing stress. American Psychologist, 44(3), 513-524.

Hui, C., Lee, C., \& Rousseau, D. M. (2004). Psychological contract and organizational citizenship behavior in China: Investigating generalizability and instrumentality. Journal of Applied Psychology, 89(2), 311-321.

Hur, W. M., Moon, T., \& Jun, J. K. (2016). The effect of workplace incivility on service employee creativity: The mediating role of emotional exhaustion and intrinsic motivation. Journal of Services Marketing, 30(3), 302-315.

Kern, J. H., \& Grandey, A. A. (2009). Customer incivility as a social stressor: The role of race and racial identity for service employees. Journal of Occupational Health Psychology, 14(1), 46-57.

Kim, H., \& Qu, H. (2018). The effects of experienced customer incivility on employees' behavior toward customers and coworkers. Journal of Hospitality \& Tourism Research, 43(1), 58-77.

Luo, P., \& Bao, Z. (2013). Affectivity, emotional exhaustion, and service sabotage behavior: The mediation role of rumination. Social Behavior and Personality: An International Journal, 41(4), 651-661.

Lustig, N., Pessino, C., \& Scott, J. (2014). The impact of taxes and social spending on inequality and poverty in Argentina, Bolivia, Brazil, Mexico, Peru, and Uruguay: Introduction to the special issue. Public Finance Review, 42(3), 287-303. 
Maslach, C., Jackson, S. E., \& Leiter, M. P. (1997). Maslach burnout inventory manual (3rd ed.). Palo Alto, CA: Consulting Psychologists Press.

Nguyen, T., \& Thien Dieu (2019). Góc khuất vệ sinh khách sạn: Tổng cục Du lịch yêu cầu kiểm tra [Hidden corner of hotel hygiene: Vietnam National Administration of Tourism requires inspection]. Truy cập ngày 15/02/2021 tại https://tuoitre.vn/goc-khuat-ve-sinh-khach-santong-cuc-du-lich-yeu-cau-kiem-tra-20190715224243767.htm

Quang Le (2019). Resort Aroma phản hồi chính thức về việc bị tố lùa đảo, đe dọa Khoa Pug: Sụ hiểu lầm trong giao tiếp không mong muốn [Resort Aroma official response to accusations of fraud and threats Khoa Pug: Misunderstanding in unwanted communication]. Truy cập ngày 15/02/2021 tại https://cafef.vn/resort-aroma-phan-hoi-chinh-thuc-ve-viec-bi-to-luadao-de-doa-khoa-pug-su-hieu-lam-trong-giao-tiep-khong-mong-muon20190407021418742.chn

Rhoades, L., \& Eisenberger, R. (2002). Perceived organizational support: A review of the literature. Journal of Applied Psychology, 87(4), 698-714.

Skarlicki, D. P., Van Jaarsveld, D. D., \& Walker, D. D. (2008). Getting even for customer mistreatment: The role of moral identity in the relationship between customer interpersonal injustice and employee sabotage. Journal of Applied Psychology, 93(6), 1335-1347.

Sliter, M., Jex, S., Wolford, K., \& McInnerney, J. (2010). How rude! Emotional labor as a mediator between customer incivility and employee outcomes. Journal of Occupational Health Psychology, 15(4), 468-481.

Sliter, M., Sliter, K., \& Jex, S. (2012). The employee as a punching bag: The effect of multiple sources of incivility on employee withdrawal behavior and sales performance. Journal of Organizational Behavior, 33(1), 121-139.

Spector, P. E., \& Fox, S. (2002). An emotion-centered model of voluntary work behavior: Some parallels between counterproductive work behavior and organizational citizenship behavior. Human Resource Management Review, 12(2), 269-292.

Thuy Linh (2017). United Airlines mất hàng trăm triệu USD vu đuổi hành khách gốc Việt [United Airlines lost hundreds of millions of dollars in evicting passengers of Vietnamese origin]. Truy cập ngày 15/02/2021 tại https://thanhnien.vn/tai-chinh-kinh-doanh/united-airlinesmat-hang-tram-trieu-usd-vu-duoi-hanh-khach-goc-viet-824941.html

Torres, E. N., Van Niekerk, M., \& Orlowski, M. (2017). Customer and employee incivility and its causal effects in the hospitality industry. Journal of Hospitality Marketing \& Management, 26(1), 48-66.

Van Jaarsveld, D. D., Walker, D. D., \& Skarlicki, D. P. (2010). The role of job demands and emotional exhaustion in the relationship between customer and employee incivility. Journal of Management, 36(6), 1486-1504.

Wang, M., Liao, H., Zhan, Y., \& Shi, J. (2011). Daily customer mistreatment and employee sabotage against customers: Examining emotion and resource perspectives. Academy of Management Journal, 54(2), 312-334.

Wang, M., Liu, S., Liao, H., Gong, Y., Kammeyer-Mueller, J., \& Shi, J. (2013). Can't get it out of my mind: Employee rumination after customer mistreatment and negative mood in the next morning. Journal of Applied Psychology, 98(6), 989-1004. 
Watts, J., Robertson, N., Winter, R., \& Leeson, D. (2013). Evaluation of organisational culture and nurse burnout. Nurs Manag (Harrow), 20(6), 24-29.

Wilson, N. L., \& Holmvall, C. M. (2013). The development and validation of the incivility from customers scale. Journal of Occupational Health Psychology, 18(3), 310-326.

Wright, T. A., \& Cropanzano, R. (1998). Emotional exhaustion as a predictor of job performance and voluntary turnover. Journal of Applied Psychology, 83(3), 486-493.

Wu, W., Changyang, H., \& Qin, Z. (2019). The influence of negative mood on organizational citizenship behavior and counterproductive work behavior: A self-control perspective. Management Review, 31(12), 146-158. 